\title{
Challenges in planning and initiating a randomized clinical study of sphincter of Oddi dysfunction
}

\author{
Peter B. Cotton, MD, FRCP, FRCS, Valerie Durkalski, PhD, MPH, Kyle B. Orrell, MSHP, \\ ACRP, Olga Brawman-Mintzer, MD, Douglas A. Drossman, MD, C. Mel Wilcox, MD, Patrick \\ D. Mauldin, PhD, Grace H. Elta, MD, Paul R. Tarnasky, MD, Evan L. Fogel, MD, MSc, \\ FRCPC, Sanjay B. Jagganath, MD, Richard A. Kozarek, MD, Martin L. Freeman, MD, Joseph \\ Romagnuolo, MD, FRCPC, MScEpid, and Patricia R. Robuck, PhD, MPH \\ Division of Gastroenterology and Hepatology (P.B.C., J.R., K.B.O.), Digestive Disease Center, \\ Division of Biometry and Epidemiology, Department of Medicine (V.D.), Anxiety Disorders \\ Program (O.B.-M.), Department of Clinical Pharmacy and Outcome Studies, South Carolina \\ College of Pharmacy (P.D.M.), Medical University of South Carolina, Charleston, South Carolina; \\ Center for Functional GI and Motility Disorders (D.A.D.), University of North Carolina, Chapel Hill, \\ North Carolina; Division of Gastroenterology (C.M.W.), University of Alabama, Birmingham, \\ Alabama; Division of Gastroenterology (G.H.E.), University of Michigan, Ann Arbor, Michigan; \\ Methodist Dallas Medical Center (P.R.T.), Dallas, Texas; Division of Gastroenterology and \\ Hepatology (E.L.F.), Indiana University Medical Center, Indianapolis, Indiana; Division of \\ Gastroenterology (S.B.J.), Johns Hopkins Hospital, Baltimore, Maryland; Virginia Mason Medical \\ Center (R.A.K.), Seattle, Washington; Division of Gastroenterology (M.L.F.), Hepatology and \\ Nutrition, University of Minnesota Medical Center, Minneapolis, Minnesota; Division of Digestive \\ Diseases and Nutrition (P.R.R.), National Institute of Diabetes and Digestive and Kidney \\ Diseases, Bethesda, Maryland.
}

\section{Abstract}

Background-Sphincter of Oddi dysfunction (SOD) is a controversial topic, especially in patients with no objective findings on laboratory or imaging studies (SOD type III). The value of ERCP manometry with sphincterotomy is unproven and carries significant risks.

\footnotetext{
Objective-To describe the process of planning and initiating a randomized sham-controlled study to establish whether patients with SOD respond to sphincter ablation, and whether the outcomes are predicted by the pain patterns, presence or absence of other functional GI or psychosocial problems, or the results of manometry.
}

Design-Planning a trial to establish which patients with "suspected SOD" (if any) respond to endoscopic sphincter ablation.

\footnotetext{
Copyright $\odot 2010$ by the American Society for Gastrointestinal Endoscopy Reprint requests: Peter B. Cotton, MD, FRCP, FRCS, Division of Gastroenterology and Hepatology, Medical University of South Carolina, 25 Courtenay Drive, ART 7100A, MSC 290, Charleston, SC $29425 .$.

DISCLOSURE: All authors disclosed no financial relationships relevant to this publication. Research support for this study was provided by the National Institute of Diabetes and Digestive and Kidney Diseases, grant U01DK074739.
} 
Setting-Meetings and correspondence by a planning group of gastroenterologists and clinical research specialists hosted at the Medical University of South Carolina.

Patients-Clarifying subject characteristics and inclusion and exclusion criteria.

Interventions-Defining the questionnaires, therapies, randomizations, and numbers of subjects required by outcome measures. Defining the metrics of success and failure.

Results-The planning resulted in funding for the proposed study as a cooperative agreement with the National Institute of Diabetes and Digestive and Kidney Diseases.

Limitations-Lack of data required several consensus decisions in designing the protocol.

Conclusion-The planning process was challenging, and some changes were needed after initiation.* (Clinical trial registration number: NCT00688662.) (Gastrointest Endosc

2010;72:986-91.)

Sphincter of Oddi dysfunction (SOD) encompasses a spectrum of disorders in which stenosis or spasm of the biliary and/or pancreatic sphincters results in episodes of abdominal pain. ${ }^{1-6}$ The diagnosis is often considered in patients who have undergone cholecystectomy. Patients with few or no laboratory or imaging abnormalities (ie, SOD type III) are very difficult to evaluate and to manage effectively. ${ }^{7}$ Imaging tests can exclude other pathologies, but there are no simple diagnostic tests for SOD. ERCP is usually considered when noninvasive diagnostic avenues have been explored and after trials of medical therapies. The purpose is to check that there are no subtle structural abnormalities in the papilla, biliary tree, or pancreas and to perform sphincter of Oddi manometry (SOM) ${ }^{2,8}$ and possible sphincterotomy. There are several major outstanding issues. Although two randomized controlled trials in patients with SOD type II have shown that the results of SOM are somewhat predictive of the outcome of biliary sphincterotomy, ${ }^{9,10}$ this has never been proven in type III patients. An important problem in this context is that ERCP (with or without SOM) carries significant risks, especially pancreatitis. ${ }^{11,12}$ Another issue is that many patients suspected of having SOD also have symptoms of more generalized digestive dysfunction (eg, irritable bowel syndrome), and problems with anxiety or depression. ${ }^{13,14}$ Whether their presence predicts the results of treatment aimed at the sphincter has not been established. Furthermore, assessment of treatment outcomes is made difficult by the known placebo response to sham interventions. ${ }^{4,5,9,10}$ All of these facts mandate the need for more research, as recommended by the latest deliberations of the Rome committee. ${ }^{15}$

We received a planning grant from the National Institute of Diabetes and Digestive and Kidney Diseases in 2004 to explore appropriate designs for a comprehensive clinical study to test the primary hypothesis that some patients with SOD respond to sphincterotomy. The EPISOD (Evaluating Predictors and Interventions in Sphincter of Oddi Dysfunction) study was funded in June 2007, and recruitment began in 6 U.S. centers late in 2008. The present commentary discusses the challenges in planning and starting the study.

\section{DEFINING THE STUDY POPULATION}

A fundamental issue was to define the study population. We used the clinical definition of SOD recently updated by the Rome III committee, ${ }^{15}$ with some points of clarification. 


\section{Gall bladder status}

Although SOD may occur in patients with intact gall bladders, attributing the pain specifically to sphincter dys-function in that context is doubly difficult. We limited the study population to patients with persistent or recurrent pain after cholecystectomy (and after $>3$ months to get baseline data).

\section{Pain burden issues}

Pain is the major symptom of SOD, and its relief is the main potential benefit of treatment. Measuring pain burden proved to be challenging. Standard pain scoring instruments ${ }^{16,17}$ are not suitable in SOD, where pains may be intermittent and vary in severity. We found an analogy in the Migraine Disability Assessment instrument developed in the context of migraine headaches. ${ }^{18}$ In collaboration with the codeveloper of the Migraine Disability Assessment, Dr Stewart, we developed and tested an analogous instrument, Recurrent Abdominal Pain Intensity and Disability (RAPID). ${ }^{19}$ By using this instrument, we defined a minimum amount of baseline abdominal pain-related disability that was considered to be sufficient to justify the risks of ERCP intervention. The eligibility criterion was set at grade 3 , which translates into $\geq 12$ days of lost time because of abdominal pain episodes from paid work or school, household work, or nonwork activities over the preceding 3 months.

Rome III included "no daily pain" as a new criterion in their definition of SOD, in an attempt to separate this syndrome from other functional GI disorders. However, many patients presenting with "suspected SOD" do have some symptoms every day as well as episodes of disabling pain, so it was agreed to allow daily "discomfort" if $<3$ in severity on a scale of 1 to 10 .

\section{Excluding structural disease}

To exclude other pathologies and overlap syndromes, subjects must have a negative upper endoscopy (and negative testing or prior treatment for Helicobacter pylori), and abdominal scans showing no structural lesion. The acceptable size of the bile duct after cholecystectomy is a controversial topic, but we settled on a diameter of $₫ \mathrm{~mm}$.

\section{Laboratory findings}

The Rome criteria for biliary SOD include the need for normal amylase and lipase, but add that elevated liver tests with pain episodes may be supportive. The goal of the study was to address the most controversial group of patients, ie, those with normal liver tests. However, slight elevations of transaminases are common, partly because of the prevalence of fatty liver disease, so the study allowed transaminases up to twice the upper limit of normal, provided that the elevation was not in the context of a pain exacerbation that normalizes afterward. The Rome criteria for pancreatic SOD state that amylase/lipase must be elevated. ${ }^{15}$ However, referral centers performing large numbers of SOM procedures commonly find elevated pancreatic sphincter pressures in patients in whom amylase/lipase have been consistently normal. 


\section{Evidence for pancreatitis}

SOD (as judged by SOM abnormality) is found in many patients with recurrent and chronic pancreatitis. ${ }^{2,5}$ We excluded anyone with a definite history of acute or chronic pancreatitis to focus on SOD as a cause of pain.

\section{Trials of medical therapy}

To ensure that simple methods had been exhausted, we required trials of acid-suppression medications and anti-spasmodics. Anyone taking antidepressants had to be on a stable dose.

\section{Other exclusion criteria}

Patients who had previously undergone any sphincter therapy or surgery involving biliary diversion were excluded from the study. Enrollment was limited also to subjects aged 18 to 65 years. Although SOD occurs in children, it is rare. In patients aged $>65$ years, there is increasing likelihood of finding other pathology, such as tumors, and of confusing comorbidities. To exclude the possibility of narcotic bowel syndrome, and to avoid the difficulties of assessing treatment responses, we excluded any patient taking narcotics on a daily basis.

\section{STUDY PROCEDURES}

\section{Prescreening}

Scheduling staff at each site reviewed referring materials to identify patients with pain and 4 basic criteria, ie, age 18 to 65 years, postcholecystectomy, and with no prior sphincter treatment or pancreatitis. These materials are then reviewed by clinical staff for the other inclusion/exclusion criteria (Table 1). Patients who appeared to be eligible were educated about the study and invited to participate. If they agreed, they signed a comprehensive consent document and then completed questionnaires to assess the presence of significant psychiatric illness and other functional digestive disorders. These issues were explored with the Mini International Neuropsychiatric Interview, ${ }^{20}$ Hospital Anxiety and Depression, ${ }^{21}$ Coping Strategies Questionnaire-Catastrophizing Subscale, ${ }^{22}$ Trauma Questionnaire-Short Form, ${ }^{23}$ Beck Depression Inventory, ${ }^{24}$ and the Short Form $36 .{ }^{25}$ If no exclusion criteria emerged, subjects were enrolled and proceeded to ERCP.

\section{ERCP}

ERCP was performed in the standard manner under conscious sedation or anesthesia. The type and amount of medications that could be given before manometry was defined, and their doses were recorded. A pancreatogram was obtained to rule out pancreas divisum (an exclusion criterion), and manometry was performed (at least for the pancreatic sphincter) by standard water-perfused technique. Abnormality was defined as a basal pressure of $>40 \mathrm{~mm}$ $\mathrm{Hg}$ in both leads, sustained for 230 seconds.

\section{Sphincterotomy allocation}

Treatment in this study meant endoscopic sphincterotomy at the time of ERCP, but there are two sphincters. Although closely related, the biliary and pancreatic sphincters can yield 
discordant readings, ${ }^{6}$ so it is necessary to clarify whether treatment is aimed at either one separately, or at both. The simplest study design to answer the question would be to have 3 arms, ie, sham, biliary sphincterotomy alone, and dual (biliary and pancreatic) sphincterotomy, regardless of the manometry results. We felt that it is ethical to allow biliary sphincterotomy in the face of normal sphincter pressures (which often occur in practice), but pancreatic sphincterotomy carries greater short-and long-term risks. We addressed this problem by a two-stage randomization. The initial allocation was to sham or sphincterotomy regardless of the manometry results. Then, those patients assigned to sphincterotomy and who were found to have elevated pancreatic sphincter pressures were randomized again to biliary sphincterotomy or to both biliary and pancreatic sphincterotomy.

Treatment allocation was done centrally by using a validated clinical trials data management system referred to as WebDCU. For the rare case that the website could not be accessed, an emergency randomization envelope was available in the endoscopy suite.

\section{Pancreatic stenting}

All patients in the study received a small (3F or 4F) temporary pancreatic stent to reduce the risk of post-procedure pancreatitis. ${ }^{12}$ These passed spontaneously over a few days or weeks, as confirmed by abdominal radiographs.

\section{Post-ERCP care, blinding, and follow-up}

Subjects were admitted for overnight observation. To avoid assessment bias, especially because the outcomes (pain severity and impact/disability) can be somewhat subjective, the EPISOD subjects and their caregivers were blinded to the treatment allocation until the end of the study. Because the ERCP was grant funded, patients did not receive a bill for their procedure. Subjects were followed by phone calls at monthly intervals for a year by a study coordinator blinded to the treatment allocation.

\section{DEFINING SUCCESS AND FAILURE}

The primary goal of the study was to see whether patients respond to sphincter ablation. This meant deciding what level of improvement constitutes success and after what time interval. Patients had to be at grade 3 disability on the RAPID instrument to enter the study. Success was defined as a RAPID grade of 1 (mildly limiting or no disability) at both 9 and 12 months after randomization. Failure was defined as a RAPID grade $>1$ at month 9 or 12 after randomization, an increase in narcotic use, or recommendation for repeated intervention.

To eliminate bias in the decisions about reintervention, each site designated an evaluating physician who was not one of the treating physicians for the study. Patients unhappy with their progress consulted with the evaluating physician, who was blinded to the treatment allocation but had all other study clinical data, including the sequential RAPID scores. The evaluating physician could recommend continuing medical treatment or referral back to a treating physician at that site for consideration of repeated ERCP and treatment. This 
recommendation defined failure, even if the treating physician disagreed and declined intervention at that time.

\section{SAMPLE SIZE}

For reasons of ethics and statistical power, and to encourage participation in the trial, a 2:1 randomization ratio was chosen in favor of sphincterotomy. This proportion of sphincterotomy to no sphincterotomy mimics the chance of getting a sphincterotomy in standard clinical practice, when the decision is made based on SOM and about two-thirds of patients have positive SOM.

Studies with sham arms have shown a $\sim 30 \%$ placebo response rate. ${ }^{4,5,18,19}$ A clinically relevant absolute difference in success rates between the two interventions (sham vs sphincterotomy) was chosen at $30 \%$ (success rate sham $=30 \%$; success rate $_{\text {sphc }}=60 \%$ ). The study was powered to assure $>90 \%$ likelihood of identifying a difference in success rates $\mathbf{2 3 0 \%}$. Sample size estimation was based on the comparison of independent proportions with a 2:1 (sphincterotomy:sham) randomization scheme. The maximum sample size required for randomization was 192 subjects (128 in treatment and 64 in sham group). Assuming a 10\% rate for subjects who drop out or are lost to follow-up, a total of 214 subjects was required for randomization. This number was increased to 250 because of anticipated loss of $15 \%$ of subjects at the time of ERCP as a result of finding pancreas divisum or failing pancreatic SOM. Thus, a total of 250 SOD III patients were to be enrolled in the study.

\section{ECONOMICS OF SOD}

SOD is a common and costly condition for which an effective early treatment may be expected to improve patients' future quality of life and to reduce the cost of medical care for their remaining lifetime. The EPISOD economic analysis will evaluate the economic impact of endoscopic sphincterotomy in patients with SOD. We will calculate incremental costeffectiveness ratios and cost increases/cost offsets caused by the differences in therapy.

\section{SAFETY ISSUES}

An independent Data and Safety Monitoring Board appointed by and advisory to the National Institute of Diabetes and Digestive and Kidney Diseases reviewed and made recommendations on the original study design and is currently monitoring the study. A Medical Safety Monitor was appointed to review serious adverse events as they occur and advise the National Institute of Diabetes and Digestive and Kidney Diseases and the Data and Safety Monitoring Board on any safety concerns that require prompt attention. In addition, serious adverse events are reported to the clinical coordinating center and the Institutional Review Board at the site where the event occurs.

Adverse events judged to be related to the ERCP procedure are documented by using a standard lexicon. ${ }^{26}$ All others occurring during the year of follow-up are defined and graded by the Common Terminology Criteria for Adverse Events system in use in cancer trials. ${ }^{27}$ The costs of any complications are not covered by the grant, and this is clearly stated in the consent process. 


\section{ANCILLARY STUDY: EPISOD 2}

Recognizing that some eligible patients may not accept randomization, a naturalistic followup study referred to as EPISOD 2 allows consenting patients to be followed for 12 months in the same way as those in the main study, after "standard" manometry-directed therapy. This will provide additional valuable information about treatment response, and also about the relationships between the response and the presence or absence of other functional and psychiatric disorders.

\section{CHALLENGES AFTER THE STUDY COMMENCED}

Approximately 12 months after the first site was initiated, 507 patients had been prescreened. Of the 87 who appeared to be eligible, 65 consented to the study. Thirty-one of these were ineligible after final screening: 25 were randomized, and 8 were entered into EPISOD 2. This inadequate accrual rate led to discussion about the reasons.

Analysis of the screen failure log showed that the 4 most common reasons for ineligibility were liver tests slightly out of range, elevations of amylase/lipase, daily pain, and daily narcotic use. After extensive discussion, the Data and Safety Monitoring Board approved some relaxation of the first 3 criteria. Liver test limits were raised to direct bilirubin and alkaline phosphatase $<2 \times$ normal, transaminases $<3 \times$ normal, and amylase/lipase to $<3 \times$ normal. The increases in the lab test limits were thought to be justified because minor elevations were common and often not sustained. The daily pain exclusion in Rome III was eliminated after discussion with the chair of the Rome project. There is no evidence that daily pain excludes the diagnosis of SOD or successful treatment, and including this category would provide useful data on those points. The daily narcotic use exclusion remained in place.

The second key issue was publicity, which has been addressed aggressively by multiple methods, including through professional organizations, brochures at meetings, letters to referring physicians, and internet advertising.

To enhance recruitment further, two more sites were added, and additional funding was approved to increase the time available for coordinators to screen proactively.

\section{Abbreviations}

RAPID Recurrent Abdominal Pain Intensity and Disability instrument

SOD sphincter of Oddi dysfunction

SOM sphincter of Oddi manometry

\section{REFERENCES}

1. Steinberg WM. Sphincter of Oddi dysfunction: a clinical controversy. Gastroenterology. 1988; 95:1409-15. [PubMed: 3049221]

2. Varadarajulu S, Hawes RH. Key issues in sphincter of Oddi dysfunction. Gastrointest Endosc Clin North Am. 2003; 13:671-94. 
3. Hogan WJ, Geenen JE. Biliary dyskinesia. Endoscopy. 1988; 20(Suppl 1):179-83. [PubMed: 3168947]

4. Petersen BT. An evidence-based review of sphincter of Oddi dysfunction: part 1, presentations with "objective" biliary findings (types I and II). Gastrointest Endosc. 2004; 59:525-34. [PubMed: 15044889]

5. Petersen BT. Sphincter of Oddi dysfunction, part 2: evidence-based review of the presentations, with "objective" pancreatic findings (types I and II) and of presumptive type III. Gastrointest Endosc. 2004; 59:670-87. [PubMed: 15114311]

6. Sherman S, Lehman GA. Sphincter of Oddi dysfunction: diagnosis and treatment. J Pancreas. 2001; 2:382-400.

7. Cohen S, Bacon BR, Berlin JA, et al. National Institutes of Health State-ofthe-Science Conference statement: ERCP for diagnosis and therapy, January 14-16, 2002. Gastrointest Endosc. 2002; 56:803-9. [PubMed: 12447289]

8. Viceconte G, Micheletti A. Endoscopic manometry of sphincter of Oddi: its usefulness for the diagnosis and treatment of benign papillary stenosis. Scand J Gastroenterol. 1995; 30:797-803. [PubMed: 7481549]

9. Geenen JE, Hogan WJ, Dodds WJ, et al. The efficacy of endoscopic sphincterotomy after cholecystectomy in patients with sphincter of Oddi dysfunction. N Engl J Med. 1989; 320:82-7. [PubMed: 2643038]

10. Toouli J, Roberts-Thomson IC, Kellow J, et al. Manometry based randomized trial of endoscopic sphincterotomy for sphincter of Oddi dysfunction. Gut. 2000; 46:98-102. [PubMed: 10601063]

11. Cotton, PB. ERCP risks, prevention and management.. In: Cotton, PB.; Leung, JWC., editors. Advanced digestive endoscopy: ERCP. Blackwell; Oxford: 2006.

12. Freeman ML, Guda NM. Prevention of post-ERCP pancreatitis: a comprehensive review. Gastrointest Endosc. 2004; 59:845-64. [PubMed: 15173799]

13. Okolo P, Sherman S, Lehman GA, et al. Psychometric profiles of patients with sphincter of Oddi dysfunction. Gastrointest Endosc. 1994; 40:119. [PubMed: 8163120]

14. Linder JD, Klapow JC, Linder SD, Wilcox CM. Incomplete response to endoscopic sphincterotomy in patients with sphincter of Oddi dysfunction: evidence for a chronic pain disorder. Am J Gastroenterol. 2003; 98:1738-43. [PubMed: 12907327]

15. Behar J, Corazziari E, Guelrud M, et al. Functional gall bladder and sphincter of Oddi disorders. Gastroenterology. 2006; 130:1498-509. [PubMed: 16678563]

16. Kane RL, Bershadsky B, Lin W-C, et al. Efforts to standardize the reporting of pain. J Clin Epidemiol. 2002; 55:105-10. [PubMed: 11809346]

17. Katz J, Melzack R. Measurement of pain. Surg Clin North Am. 1999; 79:231-52. [PubMed: 10352653]

18. Lipton RB, Stewart WF, Sawyer MB, Edmeads JG. Clinical utility of an instrument assessing migraine disability: the Migraine Disability Assessment (MIDAS) questionnaire. Headache. 2001; 41:854-61. [PubMed: 11703471]

19. Durkalski V, Stewart W, MacDougall P, et al. Measuring episodic abdominal pain and disability in suspected sphincter of Oddi dysfunction. World J Gastroenterol. 2010; 16:4416-21. [PubMed: 20845508]

20. Sheehan DV, Lecrubier Y, Sheehan KH, et al. The Mini-International Neuropsychiatric Interview (M.I.N.I.): the development and validation of a structured diagnostic psychiatric interview for DSM-IV and ICD-10. J Clin Psychiatry. 1998; 59(Suppl 20):22-33. [PubMed: 9881538]

21. Zigmond AS, Snaith RP. The Hospital Anxiety and Depression Scale. Acta Psychiatr Scand. 1983; 67:361-70. [PubMed: 6880820]

22. Keefe FJ, Williams DA. A comparison of coping strategies in chronic pain patients in different age groups. J Gerontol. 1990; 45:P161-5. [PubMed: 2365972]

23. Drossman DA, Creed FH, Olden KW, et al. Psychosocial aspects of the functional gastrointestinal disorders. Gut. 1999; 45(Suppl 2):II25-30. [PubMed: 10457041]

24. Beck, AT.; Steer, RA.; Brown, GK. Manual for the Beck Depression Inventory-II. Psychological Corp; San Antonio, TX: 1996. 
25. Ware JE, Sherbourne CD. The MOS 36-item short form Health Survey (SF-36): I. Conceptual framework and item selection. Med Care. 1992; 30:473-83. [PubMed: 1593914]

26. Cotton PB, Lehman G, Vennes J, et al. Endoscopic sphincterotomy, complications and their management. An attempt at consensus. Gastroin-test Endosc. 1991; 37:383-93.

27. Cancer Therapy Evaluation Program, National Cancer Institute. [May, 2006] National InstitutesofHealth.Commonterminologycriteriaforadverseevents.Version 3.0. Index. Available at: http://ctep.cancer.gov/protocolDevelopment/electronic_applications/docs/ ctcae_index.pdf\#search="adverseevents." 


\section{Take-home Message}

- Designing and initiating a randomized sham-controlled trial of patients with "suspected sphincter of Oddi dysfunction" after cholecystectomy required close collaboration between relevant interested endoscopists and established clinical researchers. There were particular challenges in defining the precise characteristics of subjects to be studied, and the criteria for success and failure. Initial slow recruitment led to the need for extensive publicity, some small changes in the protocol, and additional treatment sites. 


\section{TABLE 1}

\section{Eligibility criteria (protocol changes in italics)}

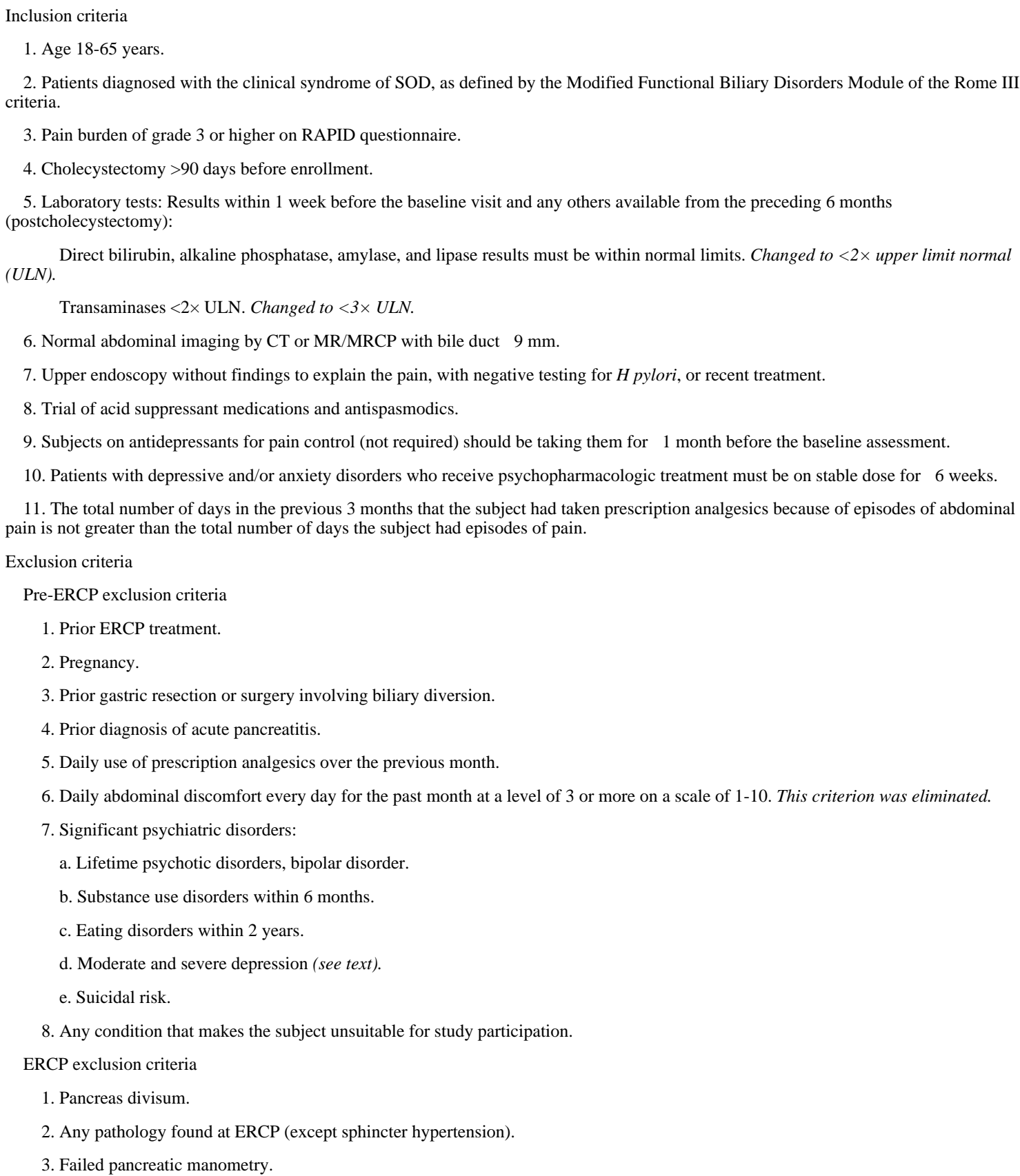

